\title{
Applying Cognitive Research to the Design of Visualization Tools: Features of the Blue Skies - College Edition Software
}

\author{
Ioanna Vekiri \& Perry Samson \\ The University of Michigan, School of Education, 610 E. University, SEB 1323, Ann Arbor, MI 48109-1259 \\ Tel: (734) 764-7469 \& (734) 763-6213, FAX: (734) 615-2164 \\ E-mail: vekiri@umich.edu, samson@umich.edu
}

\begin{abstract}
In this paper we discuss how findings from cognitive research are applied to the design of the Blue Skies: College Edition software and present findings from our current research.
\end{abstract}

Keywords: Visualization, science education, cognitive science

\section{Introduction}

Scientific visualization tools have the potential to improve science education as they can help students learn about abstract concepts and complex science processes that otherwise are difficult to study in a classroom. (Gordin \& Pea, 1995). The challenge for designers and educators is to take advantage of the possibilities of these technologies in manners that address students' needs and accommodate the way they learn. Research on the cognitive processes involved in the interpretation and use of visualizations can help us understand how to best support students' learning and interaction with symbolic representations. In this paper we discuss how findings from cognitive research are applied to the design of the Blue Skies: College Edition software and present findings from our current research.

\section{Blue Skies ${ }^{\mathrm{TM}}$ : College Edition}

Blue Skies ${ }^{\mathrm{TM}}$ : College Edition is an Internet-activated CD-ROM that is intended for college students who study meteorology. The tool enables students to explore and appreciate the dynamic world of atmospheric science using live weather information. A series of activities embedded in the software can help students visualize weather concepts and understand some of the processes and factors involved in complex weather phenomena. The activities require using and integrating information represented separately in basic weather maps and overlays (e.g. temperature, atmospheric pressure, wind speed and direction). Students are challenged to explore combinations of weather maps through a unique superimposition capability allowing one or more overlays on a basic map. This feature allows students to observe not only information about a single weather component but also patterns and relationships among various weather components (e.g. how wind direction and speed relate to atmospheric pressure differences). This approach is unique from the flat-map imagery available at many web-based weather sites as overlay fields can be included or removed interactively.

\section{Application of Cognitive Research to Software Design}

It has been proposed that graphical displays, such as diagrams, maps, and graphs, take advantage of our visual perceptual system (e.g. our tendency to configure objects into groups when they are physically close together) so as to communicate information about both individual concepts (or objects) and their relationships (Winn, 1994). This them particularly effective for helping users identify and process connections among individual pieces of information. Another advantage of graphical displays is that they are search and computationally efficient (Larkin \& Simon, 1987). In diagrams and maps all the information that users need for any cognitive task is readily available at once, which reduces the amount of information that has to be remembered (maintained in working memory) during search for all needed pieces of information.

However, research findings indicate that the search and computational efficiency of graphical displays may relate to their structural characteristics and the nature of the cognitive task for which they are used. In particular, studies based on cognitive load theory show that tasks that require learners to associate and mentally integrate 
multiple pieces of information place high cognitive demands on working memory, especially when this information comes from more than one sources (Sweller \& Chandler, 1994). Displays in which individual segments of information (e.g. a diagram and explanatory text) are separated interfere with learning and problem solving because they require students to split their attention among the different sources and therefore place an extraneous cognitive load. On the other hand, displays that provide a physical integration of all the information that is needed for a particular task in one single entity reduce cognitive load and result in better performance.

The above findings apply directly to the design of the Blue Skies: College Edition software and highlight its advantage over other tools, such as the Internet, that may provide similar resources to students who want to learn about weather phenomena. Studying atmospheric phenomena requires that students understand how various weather factors relate and interact with each other. When using weather maps, students need to integrate weather information from various maps. While students can find real-time weather maps on the Internet, they can only view one nonintegrated map at a time or view maps that are physically integrated but which may provide redundant information. Having to integrate weather information from multiple sources (maps) may interfere with students' performance as it will impose an extraneous cognitive load. On the other hand, the Blue Skies: College Edition software enables students to superimpose overlays on basic maps, and therefore to physically integrate multiple segments of information from various maps. In addition, students can select which maps they want to integrate (superimpose) depending on the task they are working on, and they do not have to process weather information that is redundant and irrelevant to that task.

\section{Research}

We conducted a pilot study to examine how the availability of the superimposition feature facilitates reasoning about weather fronts. The participants were 22 undergraduate college students who were enrolled in an introductory meteorology course and used the Blue Skies: College Edition software to complete course assignments. For the purposes of the study students were asked to use weather maps in order to locate a weather front (and draw it on a map), which is a task that requires integration of information provided in more than one weather map. The study involved two conditions (integrated and non-integrated weather maps). We found that the students who had access to the superimposition feature utilized it very often. Specifically, it was typical for this group to spend most of their time viewing combinations of maps instead of individual maps. Also, when students drew the front they used a combination of 2-4 maps, including 1 base map and 1-3 overlays. Finally, the students who used the integrated maps took more time to complete the task but used more maps in their predictions. On the other hand, the students who could view only one map at a time had to re-check the same maps several times before making their decision, more often that the students in the experimental condition $(\mathrm{p}<0.5)$. Also, the control students were less confident when they drew the front. This was indicated by the fact that more than half of them expressed the need to view some of the same maps again after they had already decided that they were ready to draw the front. This indicates that the non-integrated maps (which lack the superimposition feature) placed higher processing demands on the students and made it harder for them to remember and integrate individual pieces of weather information. The study suggests that the availability of the superimposition feature may have a positive influence on how students perform on tasks that require integration of information from various displays. In our future research we aim to investigate how other factors, such as prior knowledge, visuospatial ability, and strategies may affect students' performance in the two conditions.

\section{References}

Gordin, D. \& Pea, R.D. (1995). Prospects of scientific visualization as an educational technology. The Journal of the Learning Sciences, 4 (3), 249-279.

Larkin, J. \& Simon, H. A. (1987). Why a diagram is (sometimes) worth ten thousand words. Cognitive Science, $11,65-99$.

Sweller, J. \& Chandlre, P. (1994). Why some material is difficult to learn. Cognition and Instruction, 12 (3), 185233.

Winn, W. (1994). Contributions of perceptual and cognitive processes to the comprehension of graphics. In W. Schnotz \& R. W. Kulhavy (Eds.) Comprehension of Graphics. Amsterdam: North-Holland. 